and 2 satellites. About twenty observing stations scattered throughout the member countries are regularly making photographic observations of the two satellites so that knowledge of the size and shape of Europe may be improved. 'The camera stations photograph one of the satellites against the star background and, when this is achieved simultaneously at two or more stations, the rays to the satellite from the cameras form the sides of a triangle or a polyhedron from which it is possible to deduce something about the relative positions of the stations. For example, at about 22.25 U.T. on April 16 this year, the cameras situated at Graz in Austria, Meudon and Strasbourg in France, Frankfurt and Munich in western Germany, San Fernando in Spain and Malvern in England all photographed the Echo 1 satellite. Observations such as this establish strong geodetic ties between the respective camera stations. The observations need to be extremely accurate, about a millisecond in time and a few seconds of arc in position, if any improvement is to be made on the existing knowledge of the shape of Europe.

Photographic observations of satellites can only provide an angular measurement, and, in order to determine the distances between the stations, a scale must be introduced into the system. This can be done by assuming that the distance between two stations of the network is known or by measuring the distance of the satellite directly. One method of ranging a satellite is by the use of a pulsed laser and work in this field is being actively pursued in the United States and France. At a conference on Satellite Geodesy in Paris in May and at the recent COSPAR meeting in London, American and French workers estimated that accuracies of a few metres in the measurement of range are now possible by the use of lasers, although this is, of course, restricted to satellites which carry specially constructed corner reflectors. Eventually, an accuracy of about 10 metres will be possible for the position of each of the camera sites. Because each site can be carefully placed within a national geodetic datum, the relative positions of any two places in Europe should be determined with comparable precision.

This programme of observations of the Echo satellites, organized by the Western European Sub-Commission of the International Commission for Artificial Satellites of the International Association of Geodesy, is not the only one which has been carried out recently in Europe. The United States Coast and Geodetic Survey has used portable BC4 cameras to establish sites at several points in Europe, including Edinburgh in Scotland and Munich in western Germany. These sites are occupied for a few weeks while observations are being made and the cameras then move to another location. This European programme is part of a larger world wide triangulation programme of the United States Coast and Geodetic Survey. In addition, the Smithsonian Astrophysical Observatory has camera stations at San Fernando and Athens which are actively participating in a geodetic programme. The National Aeronautics and Space Administration is also undertaking geodetic studies which include observations from Europe. The Geos $A$ flashing light satellite has also been observed on several occasions from European camera stations, particularly Zimmerwald in Switzerland and Malvern in England. In general, satellite geodesy in Europe seems to be growing well on the observational side; the great need now is for work on the analysis of observations so as to make more widely available the results of what has been done.

\section{Meteor Showers Ahead}

\section{from a Correspondent}

THE two important meteor showers of November and December-the Leonids and the Geminids-are markedly dissimilar in character. The Geminid shower provides one of the most reliable meteor displays, with only statistical variations in the rate from year to year, but the Leonids are typical of another type of stream in which remarkable showers occur periodically, indicating an intense localized swarm of particles in the orbit.

Some Geminid meteors can usually be seen between December 7 and 15, but the maximum occurs on December 13 or 14, when an observer may see as many as fifty meteors an hour with the naked eye if the sky conditions are good. Thus the Geminids are one of the richest showers of the year. Oddly enough, however, there are no records of a shower on these dates in antiquity, and the shower does not seem to have appeared with sufficient strength to be recognized until 1862.

Its origin is an enigma. The formation of meteor streams is now generally supposed to be a result of the disintegration of comets as they swing around the Sun. In favour of this view, several meteor streams are known to move in the same orbits as certain comets -in fact, one of the first cometary associations to be demonstrated was that of the Leonids and Tempel's comet. The Geminids have never been satisfactorily accounted for by this hypothesis. Their orbit is peculiarly small, smaller than that of any known meteor stream or comet and of most asteroids. Geminid meteors are also unique in having densities two to three times greater than the average density of meteoroids. The explanation of these abnormalities by an old parent comet in which the meteoric dust is likely to be more compact, coupled with perturbations of the orbit by close approaches to the planets, cannot be ruled out, but the exact mechanism remains a mystery.

Interest in the Leonids, which flagged somewhat as a result of the disappointing maxima of 1899 and 1932 , has revived after the historic display over North America in 1966. Last year the Soviet astronomer I. S. Astapovich predicted that the maximum of the 1966 Leonid shower would occur on November 17 at 10.00 U.T.- a forecast which was very nearly correct. Observing stations in northern Siberia, the only region of the Soviet Union where the radiant was above the horizon during darkness, recorded maximum activity between 11.50 and 12.30 U.T. on November 17 , and a zenithal hourly rate at peak activity of 130,000 , in close agreement with observations in the United States. This year, Russian astronomers are expecting the maximum to occur on November 17 at 17.00 U.T. No predictions are being made of the strength of the shower, and perhaps astronomers are remembering that the failure of the shower to return as predicted in 1899 was, in the words of the American meteor physicist C. P. Olivier, "the worst blow ever suffered by astronomy in the eyes of the public". 